\title{
REVISED A global repository of novel antimicrobial emergence
}

\section{events [version 2; peer review: 1 approved, 2 approved with}

\section{reservations]}

Previously titled: Extracting novel antimicrobial emergence events from scientific literature and medical reports

\section{Emma Mendelsohn (D)1, Noam Ross (D1, Allison M. White 1,2, Karissa Whiting 1,3, Cale Basaraba1,4, Brooke Watson Madubuonwu1,5, Erica Johnson 1,6, Mushtaq Dualeh1,7, Zach Matson 1,7, Sonia Dattaray 1,8, Nchedochukwu Ezeokoli (i)1,9, Melanie Kirshenbaum Lieberman1,10, Jacob Kotcher1,11, Samantha Maher (iD1, Carlos Zambrana-Torrelio (iD1, Peter Daszak 1}

\footnotetext{
${ }^{1}$ EcoHealth Alliance, New York, NY, 10018, USA

${ }^{2}$ Current Address: Office of U.S. Foreign Disaster Assistance, USAID, District of Columbia, USA

${ }^{3}$ Current Address: Department of Epidemiology and Biostatistics, Memorial Sloan Kettering Cancer Center, New York, NY, USA

${ }^{4}$ Current Address: Mailman School of Public Health, Columbia University, New York, NY, USA

${ }^{5}$ Current Address: American Civil Liberties Union, New York, NY, USA

${ }^{6}$ Current Address: Biology Department, Graduate Center of the City University of New York, New York, NY, USA

${ }^{7}$ Current Address: Oak Ridge Institute for Science and Education Fellow, Centers for Disease Control and Prevention, Atlanta, GA, USA

${ }^{8}$ Current Address: Health Union, Philadelphia, PA, USA

${ }^{9}$ Current Address: Guidehouse, Chicago, IL, USA

${ }^{10}$ Current Address: School of Veterinary Medicine, University of Pennsylvania, Philadelphia, PA, USA

${ }^{11}$ Current Address: Department of Geoscience, Hobart and William Smith Colleges, Geneva, NY, USA
}

V2 First published: 12 Nov 2020, 9:1320

https://doi.org/10.12688/f1000research.26870.1

Latest published: 21 Jun 2021, 9:1320

https://doi.org/10.12688/f1000research.26870.2

\section{Abstract}

Despite considerable global surveillance of antimicrobial resistance (AMR), data on the global emergence of new resistance genotypes in bacteria has not been systematically compiled. We conducted a study of English-language scientific literature (2006-2017) and ProMED-mail disease surveillance reports (1994-2017) to identify global events of novel AMR emergence (first clinical reports of unique drug-bacteria resistance combinations). We screened 24,966 abstracts and reports, ultimately identifying 1,757 novel AMR emergence events from 268 peer-reviewed studies and 26 disease surveillance reports (294 total). Events were reported in 66 countries, with most events in the United States (152), China (128), and India (127). The most common bacteria

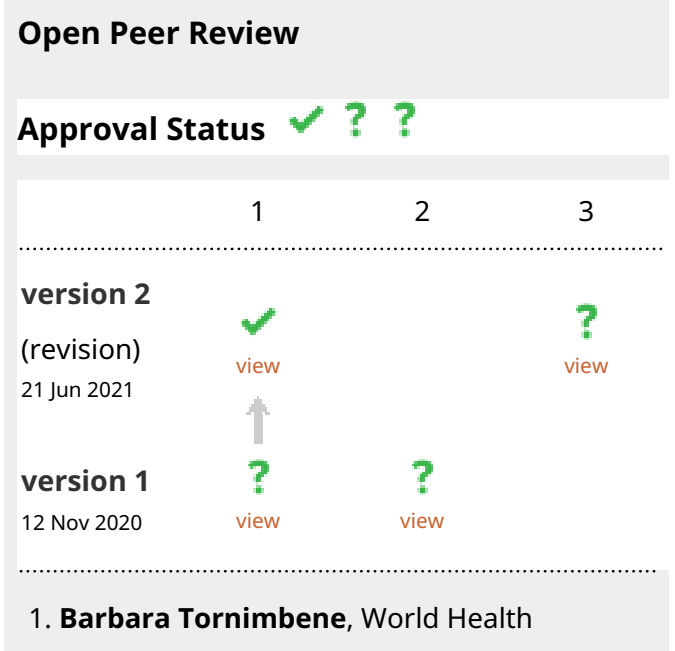

Page 1 of 22 
demonstrating new resistance were Klebsiella pneumoniae (344) and Escherichia coli (218). Resistance was most common against antibiotic drugs imipenem (89 events), ciprofloxacin (84) and ceftazidime (83). We provide an open-access database of emergence events with standardized fields for bacterial species, drugs, location, and date. We discuss the impact of reporting and surveillance bias on database coverage, and we suggest guidelines for data analysis. This database may be broadly useful for understanding rates and patterns of AMR evolution, identifying global drivers and correlates, and targeting surveillance and interventions.

\section{Keywords}

Antimicrobial resistance, global health, open-access data

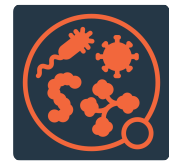

This article is included in the Pathogens gateway.

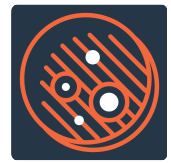

This article is included in the Antimicrobial Resistance collection.
Organization, Geneva, Switzerland

Sergey Eremin iD, World Health

Organization, Geneva, Switzerland

2. Ellen Stobberingh, Maastricht University Medical Centre (MUMC), Maastricht, The Netherlands

3. Jorge Pinto Ferreira (D), World Organisation for Animal Health, Paris, France

Any reports and responses or comments on the article can be found at the end of the article.

Corresponding authors: Emma Mendelsohn (mendelsohn@ecohealthalliance.org), Peter Daszak (daszak@ecohealthalliance.org)

Author roles: Mendelsohn E: Conceptualization, Data Curation, Methodology, Project Administration, Software, Supervision, Validation, Visualization, Writing - Original Draft Preparation, Writing - Review \& Editing; Ross N: Conceptualization, Data Curation, Methodology, Project Administration, Supervision, Validation, Writing - Original Draft Preparation, Writing - Review \& Editing; White AM: Conceptualization, Data Curation, Methodology, Project Administration, Supervision, Validation, Writing - Original Draft Preparation; Whiting K: Conceptualization, Data Curation, Methodology, Software, Validation, Writing - Original Draft Preparation; Basaraba C: Conceptualization, Data Curation, Methodology, Software, Validation; Watson Madubuonwu B: Conceptualization, Data Curation, Methodology, Validation, Writing - Original Draft Preparation; Johnson E: Conceptualization, Data Curation, Methodology, Validation, Writing - Original Draft Preparation; Dualeh M: Data Curation; Matson Z: Data Curation; Dattaray S: Data Curation, Project Administration; Ezeokoli N: Data Curation; Kirshenbaum Lieberman M: Data Curation; Kotcher J: Data Curation; Maher S: Data Curation; Zambrana-Torrelio C: Conceptualization, Methodology, Writing - Review \& Editing; Daszak P: Conceptualization, Methodology Competing interests: No competing interests were disclosed.

Grant information: United States Agency for International Development (USAID) Emerging Pandemic Threats PREDICT (Cooperative Agreement No. AID-OAA-A-14-00102).

The funders had no role in study design, data collection and analysis, decision to publish, or preparation of the manuscript.

Copyright: $\odot 2021$ Mendelsohn E et al. This is an open access article distributed under the terms of the Creative Commons Attribution License, which permits unrestricted use, distribution, and reproduction in any medium, provided the original work is properly cited.

How to cite this article: Mendelsohn E, Ross N, White AM et al. A global repository of novel antimicrobial emergence events [version 2; peer review: 1 approved, 2 approved with reservations] F1000Research 2021, 9:1320

https://doi.org/10.12688/f1000research.26870.2

First published: 12 Nov 2020, 9:1320 https://doi.org/10.12688/f1000research.26870.1 


\section{REVISED Amendments from Version 1}

In response to peer review, we have made the following edits: disaggregated all drug combinations (resulting in minor changes to reported AMR event counts); developed an alternate version of the database using the Anatomical Therapeutic Chemical (ATC) drug ontology; reconfigured Figure 1 to include more information about the database building process; updated Figure 4; addressed uncertainty related to data from ProMEDMail vs. from peer-reviewed journals; and provided clarification and rewording throughout.

Any further responses from the reviewers can be found at the end of the article

\section{Introduction}

Antimicrobial resistance (AMR) is a global health crisis that has compromised the effective treatment and prevention of a multitude of infections. The rise in AMR has been associated with increased mortality, longer hospitalizations, complications with medical procedures such as surgery and chemotherapy, and higher healthcare $\operatorname{costs}^{1-3}$. Resistance to antibiotics is a global public health issue and a particular concern in low- and middle-income countries, where many high-burden diseases such as malaria, respiratory infections, and tuberculosis can no longer be treated by common antimicrobial drugs ${ }^{1,4}$. Combating AMR requires a multidimensional global response to optimize antimicrobial drug use, improve awareness, increase traceability and usage reporting, and promote research ${ }^{1,5-7}$.

AMR surveillance by researchers, hospital networks, and state governments is key to characterizing and responding to the crisis. Current global-scale datasets primarily focus on the presence or prevalence of known resistance genotypes and phenotypes in bacterial populations. In 2014, the World Health Organization (WHO) published surveillance data obtained from 129 member states on nine bacterial pathogen-antibacterial drug combinations of public health importance, finding high rates of resistance reported across the globe ${ }^{3}$. The ResistanceMap database, maintained by the Center of Disease Dynamics, Economics \& Policy, provides nationally-aggregated data from 46 countries on the prevalence of resistance in 12 bacterial species against 17 classes of antibiotics ${ }^{8}$.

To our knowledge, however, there is no publicly available dataset that specifically identifies the spatial and temporal patterns of the emergence of novel bacterial pathogen resistance to antibiotic drugs in humans. Such data are critical to understanding macroecological patterns and drivers of AMR emergence and identifying geographic and phenotypic targets for surveillance, research, and interventions. This repository is relevant to the development of country-specific National Action Plans (NAPs) to reduce the emergence and spread of AMR, as well as to on-going agreements, programs and priorities for intergovernmental organizations (e.g., Tripartite-Plus Alliance on AMR, FAO, OIE, UNEP and WHO) and multi-lateral development banks and financing facilities (e.g. World Bank). In addition, a better understanding of factors that contribute to AMR emergence may bolster the work of the Interagency
Coordination Group (IACG) on Antimicrobial Resistance (convened by the UN Secretary General) to target drivers of AMR that are important for orientation and collective action?

We conducted a systematic review to create a repository of novel AMR emergence events reported in English-language scientific literature from 2006-2017 and ProMED-mail disease surveillance reports from 1994-2017. We focused specifically on human clinical cases and did not limit the search to any subset of bacteria or drugs. We screened 24,966 abstracts and reports, identifying 1,791 articles potentially reporting novel emergence events, and ultimately, 294 relevant articles reporting 1,757 total novel events. We present an open-access database of these events with cleaned and standardized fields for location of emergence, bacterial species, antimicrobial drug, event start date, and data source. We provide usage notes on systematic biases and ambiguities in the data. In addition, we provide data on all screened and processed articles from which data were harvested.

\section{Methods}

Development of the AMR emergence database was a multistep process consisting of a systematic literature search, abstract and report screening, article review and coding, and data cleaning and standardization (Figure 1).

\section{Literature search}

We drew from PubMed and Embase scientific literature databases and ProMED-mail to develop our database of events. PubMed and Embase collectively encompass a large fraction of peer-reviewed, English-language biomedical scientific literature, while ProMED-mail consists of clinical reports and news alerts that have a faster reporting speed and may not ultimately be published in the scientific press. We did not assess quality of studies or reports; however, we indicate in the database whether an event is from a 'peer-reviewed study' or 'ProMED-mail report'.

We searched for a recent ten-year period for scientific literature (2006-2017). ProMED-mail reports were drawn from a longer time span (1994-2017), as we found scientific articles published 2006-2017 frequently described events occurring many years before.

We searched for manuscripts in PubMed and Embase using the following terms:

'antibiotic resistance'lexp OR ('antibiotic' AND ('resistant' $O R$ 'resistance')) $O R$ 'antimicrobial resistance'/exp OR ('antimicrobial' AND ('resistant' OR 'resistance')) AND (first OR novel OR new OR emerging OR emergent) AND (case OR patient) AND [humans]/lim AND [2006-2017]/py

This search, completed August 2018, yielded 23,770 results.

Because ProMED-mail searches use partial word stem-based matching, we used only the search terms 'antibiotic resistance, and 'antimicrobial resistance', which yielded 1,196 results. 


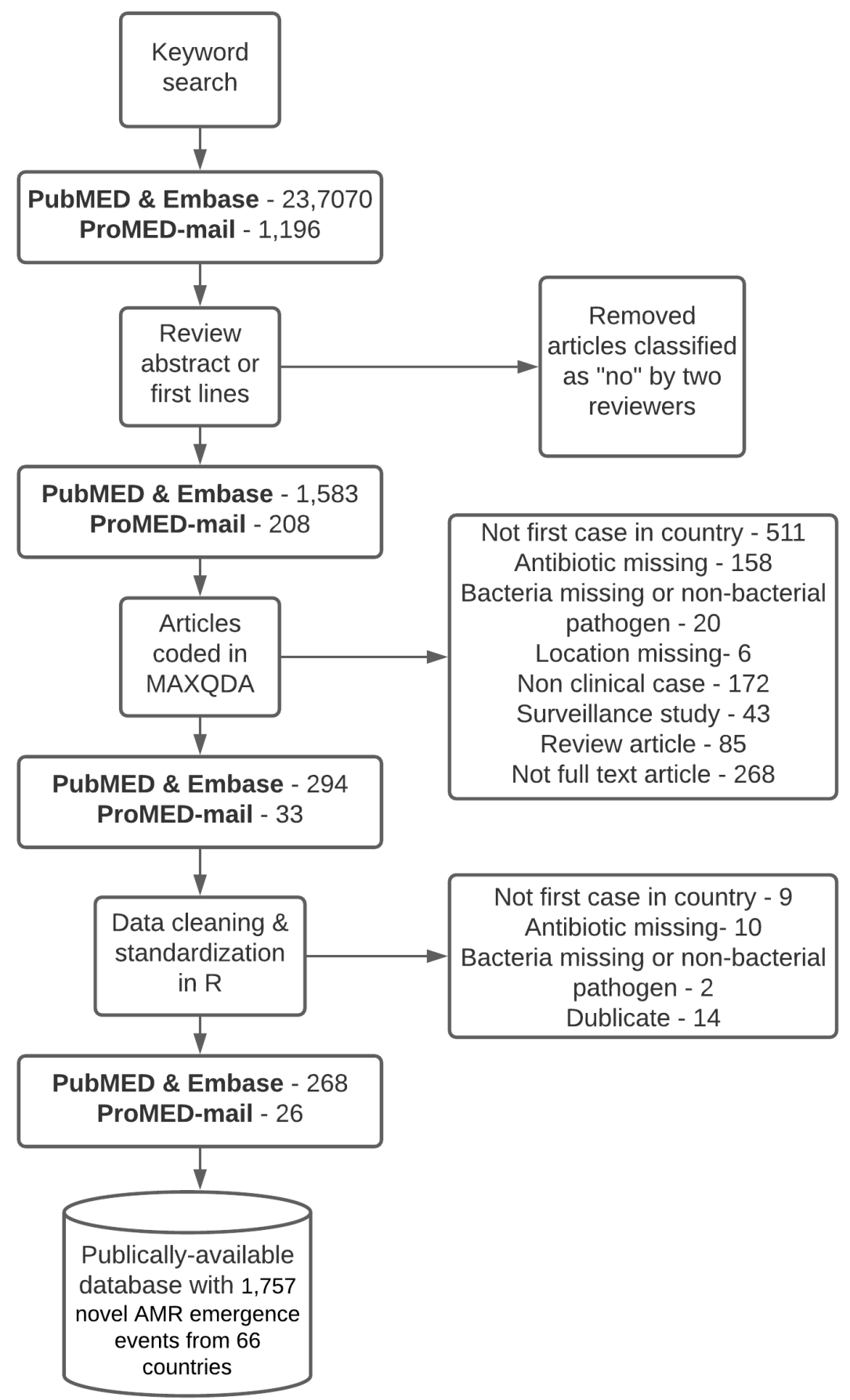

Figure 1. Prisma flow diagram, showing the workflow of information through steps of the systematic review. Note some articles were excluded based on more than one criteria.

A total of 24,966 articles (peer-reviewed studies and ProMED-mail reports) were compiled for screening.

\section{Abstract and report screening}

We used the following inclusion criteria to screen the results of our literature search: the article must have described at least one clinical case (a) of infectious disease in a patient (b) caused by a novel (c) resistance (d) to a particular antimicrobial drug or drug combination (e) in bacteria (f). In this definition:

a) A "clinical case" is an individual who presents with symptoms to a medical professional and is determined by a medical professional to have been infected with 
the bacterium species reported. Antimicrobial resistance in an asymptomatic individual's commensal bacteria-as determined by a screening study, a "challenge" study, or a laboratory trial-was excluded from review, as there is higher variability in study design among such studies than in clinical case reports.

b) The "patient" from which the bacterium of interest is identified must be human and may be of any age or gender. More than one patient can be included in an emergence event if all patients fell ill and were confirmed to be infected with a resistant bacterium at the same time, for example in the early stages of an outbreak.

c) "Novel" indicates that resistance to a given antimicrobial treatment in the bacterial species in question has not previously been detected or described in the country in question. In this definition, new mechanisms of resistance of a given bacteria to a given antimicrobial combination do not count as novel resistance (e.g. a novel plasmid carrying beta-lactamase in a bacterial species with previously described beta-lactam resistance).

d) "Resistance" is the ability of the bacteria in question to survive standard antibacterial treatment against it. Survival is measured by the bacteria's ability to continue to cause disease in its host or to spread to others for longer than the standard period following treatment. Standard treatments may be determined by the WHO or by the country in which the resistance event occurred (we deferred to the authors' selection of standard treatment protocol).

e) An "antimicrobial drug or drug combination" is a drug, drug class, or specific combination of drugs used to treat bacterial infections in humans. We focused exclusively on antibiotic resistance rather than resistance to other antimicrobials due to better data availability and based on the hypothesis that drivers for other antimicrobials might be different than for antibiotics.

f) "Bacteria" is a microorganism that may cause a disease in the domain Bacteria. We did not include cases of resistance in viruses, fungi, or other pathogen types.

For results from the PubMed and Embase search, we programmatically downloaded abstracts and metadata. Article abstracts were manually reviewed by a team of screeners (all authors, see Author contributions) to determine whether they likely contained a report of a novel emergence event, according to the above criteria. To ensure uniformity in abstract evaluation, all reviewers received training on the inclusion criteria and were required to achieve $90 \%$ agreement with a practice set of 100 previously-screened abstracts. Abstracts were screened separately by two individuals. Reviewers classified articles as "yes", "maybe", or "no" for inclusion. If both reviewers classified an article as "yes" or "maybe", the article was downloaded as full-text for further review and to be coded for the database. In cases when an article was marked as "no" by one of the reviewers, and "yes" by the other reviewer, a third reviewer was assigned to determine if the article should be included. The inter-scorer agreement rate was $82 \%$ (i.e., $18 \%$ of articles were decided by a third reviewer). 1,583 articles from PubMed and Embase passed review criteria and were downloaded for further review. The $\mathrm{R}$ package metagear ${ }^{9}$ was used to screen articles and manage screening data.

Full texts were downloaded for ProMED-mail search results, as ProMED-mail reports do not have abstracts. The first lines of each report were screened separately by two reviewers. If either reviewer classified a text as "yes" or "maybe", it was selected for further review and to be coded for the database. 208 ProMED-mail texts passed this screening, contributing to a total of 1,791 total articles from PubMed, Embase, and ProMED-mail selected for review. Further details on the reproducible screening workflow are available in the data repository (see Data availability) ${ }^{10}$.

\section{Article coding}

Articles from PubMed, Embase and ProMED-mail that passed screening were selected for full-text analysis, each by one reviewer, unless quality assurance checks required follow-up review (see Data cleaning). Reviewers read full-text articles to determine whether they fully met the case criteria, above, and to extract data by coding the text. Articles were excluded at this stage if it was found that they referred to or were duplicate reports of a previous emergence of the same drug-bacteria resistance within the country, if they reported on a non-bacterial pathogen, or if they did not identify the drug or bacterial species. A total of 294 articles were retained after full-text screening.

Articles were coded for four required fields: study country, drug name, bacteria, and event start date. Drugs were coded to the lowest available taxonomic rank (i.e., specific drug rather than drug class, when available). Similarly, bacteria were coded at the species level when available. Where articles did not include an emergence location or start date, location was inferred from the location of study authors' institutions (often hospitals), and publication year was used for start date.

In addition to the required fields, articles were coded for the following secondary fields when available: patient attributes (age, gender, country of residence, recent travel locations, symptoms, comorbidities, and outcome), bacterial strains and markers, drug minimum inhibitory concentration (MIC) values, and hospital location (city, state/province). Screeners used MAXQDA $^{11}$ software for article coding. To our knowledge, open-source equivalents to MAXQDA are not available; however, open-source software such as qcoder ${ }^{12}$ could be used to replicate MAXQDA if combined with suitable PDF pre-processing steps.

\section{Data cleaning}

We matched free-form values coded in article text to standardized values and ontologies. All locations were matched to Google Places names and geocoded. Country names were 
maintained according to article reporting and were not standardized to official country recognitions (e.g., United Nations member states). Dates were stored using the international standard ISO 8601. Drug names and bacteria were matched and standardized against the Medical Subject Headings $(\mathrm{MeSH})^{13}$ and National Center for Biotechnology Information (NCBI) Organismal Classification ${ }^{14}$ ontologies, respectively, as provided by the Bioportal platform ${ }^{15}$. We also explored standardizing drug names to the Anatomical Therapeutic Chemical (ATC) ontology, and provide this as an alternative classification $^{16}$. (We found that ATC classification resulted in similar quantitative results to MeSH-based classification; for example, there are 1,753 events in the ATC-based database versus 1,757 in the MeSH-based database.) Where reported names did not exactly match ontologies or had ambiguous matches, we manually reviewed and corrected names to match the ontologies, in some cases requiring review of the original study to confirm accuracy. Other fields that are not essential to defining emergence events (e.g., patient demographics, comorbidities) were not standardized in the current database release.

Data cleaning and standardization was performed in $\mathrm{R}$ version 3.6.1 ${ }^{17}$, using the tidyverse framework ${ }^{18}$ for data manipulation. Geocoding used the Google Geocoding API via the ggmap $\mathrm{R}$ package ${ }^{19}$. Dates were standardized using the lubridate R package ${ }^{20}$.

We implemented quality assurance checks throughout the data processing pipeline. We checked for errors in the MAXQDA article coding by confirming that all values were labeled and that links between values were properly assigned (e.g., links between drug names and MIC values). We checked for any studies missing study location, event start date, drug, or bacteria, and manually revisited these articles to confirm missing fields. We also investigated any study reporting more than one location and/or start date to confirm whether the study described multiple emergence events.

An earlier version of this article can be found on medRxiv (doi: https://doi.org/10.1101/2020.08.13.20165852).

\section{Results/Discussion}

We present a database of 1,757 records of first clinical reports of unique bacterial-drug AMR detections by country, ranging from 1998 to 2017, drawn from 268 peer-reviewed articles and 26 disease surveillance reports (294 total). (While the ProMED-mail search extended to 1994, the first reported event that met our study criteria occurred in 1998.) ProMED-mail reports had similar coverage to the peer-reviewed articles, with events reported in six continents from 1999 through 2016. This database-when used with sufficient consideration of reporting biases (see Database usage notes)—can serve as a complement to existing databases that track resistance and spread to support researchers in targeting efforts for surveillance and interventions and analyzing factors that contribute to AMR emergence.
Database materials are available at DOI 10.5281/zenodo.4924992 (see Data availability) ${ }^{10}$. The database can be downloaded as 'events-db.csv'. Field names and descriptions from the database are detailed in Table 1. The file 'data-processed/articles-db.csv' contains metadata about each article in the database, including citation information and full abstracts. This file can be joined with `events-db.csv` by the `study_id` field.

In addition to the repository, we present intermediate data used in the process of database development in our GitHub repository \{ https://github.com/ecohealthalliance/amr-db \}. All abstracts and ProMED-mail reports that were screened are in the 'screening' directory. Raw exports from coded fulltext articles are in .xlsx form in 'data-raw/coded-segments'. (The coded full text articles themselves are not available in the data repository.) A pre-filtered, pre-transformed database that contains all fields (primary and secondary) and events (including some non-emergent events) is in 'data-processed/segments.csv`. An alternative version of the database with drug names standardized to the ATC ontology is available in the 'alt-db-atc/ directory, and a one-to-one comparison of $\mathrm{MeSH}$ and ATC drug names from the database is available in 'figures-and-tables/mesh-atc-comparison.csv'. Further details on directory structure and usage are provided in the data repository documentation.

We identified AMR emergence events in 66 countries, with the most reported events in the United States (152), China (128), and India (127). (Figure 2). Events were reported from 1998 through 2017, with the greatest number of reported events occurring in 2011 (Figure 3). See Database usage notes for discussion on the effects of reporting bias on the spatial and temporal coverage of the database.

We found that Klebsiella pneumoniae and Escherichia coli were the most common bacteria in emergence events (Figure 4), supporting results from other databases that have found high rates of AMR prevalence for these species $^{3,8}$. Of concern, our database indicates that both bacteria species had the greatest number of reports of novel resistance to imipenem and meropenem (Figure 4), which are carbapenem antibiotics that are considered critical for treating infections acquired in health care settings $\mathrm{s}^{3,21,22}$. Also concerning were 23 cases of emergent resistance to the antibiotic colistin in 16 countries and 14 distinct bacteria, including $K$. pneumoniae and E. coli. Colistin is critically important for treating infections when no other options are available 22,23 .

Further analysis is needed to understand the underlying causes of AMR emergence, including the relative contributions of human and livestock antibiotic consumption. In addition to antibiotic consumption, risk factors for emergence may include human travel and migration; country population, GDP, and spending on healthcare; and production of antibiotics. Identifying risk factors for AMR emergence will support improved AMR surveillance and interventions and will align with a "One Health" approach of linking drivers from the human, animal, and environmental sectors ${ }^{24}$. 
Table 1. Database fields and descriptions.

\begin{tabular}{|c|c|}
\hline Field & Description \\
\hline ‘study_id’ & Unique study identification number that can be joined with `articles_db` for study metadata \\
\hline ‘study_country & $\begin{array}{l}\text { Name of country where event occurred. Note that there are some studies that report on events } \\
\text { in multiple countries. }\end{array}$ \\
\hline 'study_iso3c' & Three letter International Organization for Standardization (ISO) code \\
\hline ‘study_location' & Full study location (including hospital, city, and state if available) \\
\hline ‘study_location_basis' & Spatial basis of study location (e.g., "hospital, city, state_province_district, country") \\
\hline 'residence_location` & Location of patient residence \\
\hline ‘travel_location` & Patient travel locations, if any reported. Multiple locations are separated by $\dddot{i}$. \\
\hline ‘drug' & Antimicrobial drug, standardized to the Medical Subject Headings (MeSH) ontology ${ }^{13}$. \\
\hline 'drug_rank’ & Taxonomic classification of drug (i.e., drug name or group) \\
\hline 'drug_parent_name' & $\begin{array}{l}\text { Name of the taxonomic parent of antimicrobial drug, standardized to the Medical Subject } \\
\text { Headings (MeSH) ontology }{ }^{13} \text {. }\end{array}$ \\
\hline ‘bacteria` & Name of resistant bacteria, standardized to NCBI Organismal Classification ontology ${ }^{14}$. \\
\hline 'bacteria_rank’ & Taxonomic classification of bacteria name (e.g., "species", "genus") \\
\hline 'bacteria_parent_name’ & $\begin{array}{l}\text { Name of the taxonomic parent of bacteria, standardized to NCBI Organismal Classification } \\
\text { ontology }{ }^{14} \text {. }\end{array}$ \\
\hline 'bacteria_parent_rank’ & Taxonomic classification of bacteria parent name (e.g., "species", "genus") \\
\hline ‘start_date` & Date that patient was presented to hospital in format of $y y y y-m m-d d$ \\
\hline `start_date_rank’ & Specificity of the start date (i.e., year, month, day) \\
\hline ‘end_date` & Date that patient was released from hospital or died in format of yyyy-mm-dd \\
\hline 'data_source' & Whether data source is 'peer-reviewed study' or 'promed-mail report' \\
\hline
\end{tabular}

Information in this repository can be used to inform international initiatives aimed to reduce and prevent the emergence of AMR. For example, the WHO Global Action Plan on AMR articulates the need for country capacity to detect emergence of resistance ${ }^{1}$. International organizations are developing tracking systems that seek to enhance surveillance and reporting at the global level (e.g., the Global Antimicrobial Resistance and Use Surveillance System [GLASS]). Long-term, the AMR digital detection interface launched in 2020 through the Program for Monitoring Emerging Diseases (ProMED) may collate future emergence events and can consider antimicrobial use and other relevant metadata examined in this study to support trend analysis ${ }^{25}$. Finally, improved attention to AMR emergence is in line with the prevention-oriented focus of the Action Package on AMR under the Global Health Security Agenda, a partnership of $70+$ countries working to strengthen capacities to make the world safe against infectious disease threats.

\section{Database usage notes}

The database is developed using only English-language scientific literature and medical reporting events. It therefore represents events reported in this limited subset rather than truly representative clinical cases and strongly reflects reporting effort and practices. It is likely that AMR emergence events are systematically missing from the database for countries that are not English speaking and/or have less health care monitoring and reporting capacity, including those with lower GDP. Therefore, it is imperative that any analysis of the data account for the effects of reporting bias ${ }^{26}$. In addition, future efforts to characterize AMR emergence may consider other sources of data including gray literature and government reports. These sources of data may be particularly useful for informing fine-scale data needs for country decision making.

Our review did not include any quality assessments of the published scientific literature and disease surveillance reports. Data sourced from the peer-reviewed literature has gone through a process of validation and quality checked by the journal editors and reviewers. ProMED-mail reports, which are generated in real-time, are screened by regional moderators to ensure data validity, but no peer-review or editorial process is involved. This is a potential source of uncertainty in our database, and is indicated in the column 'data_source' . 


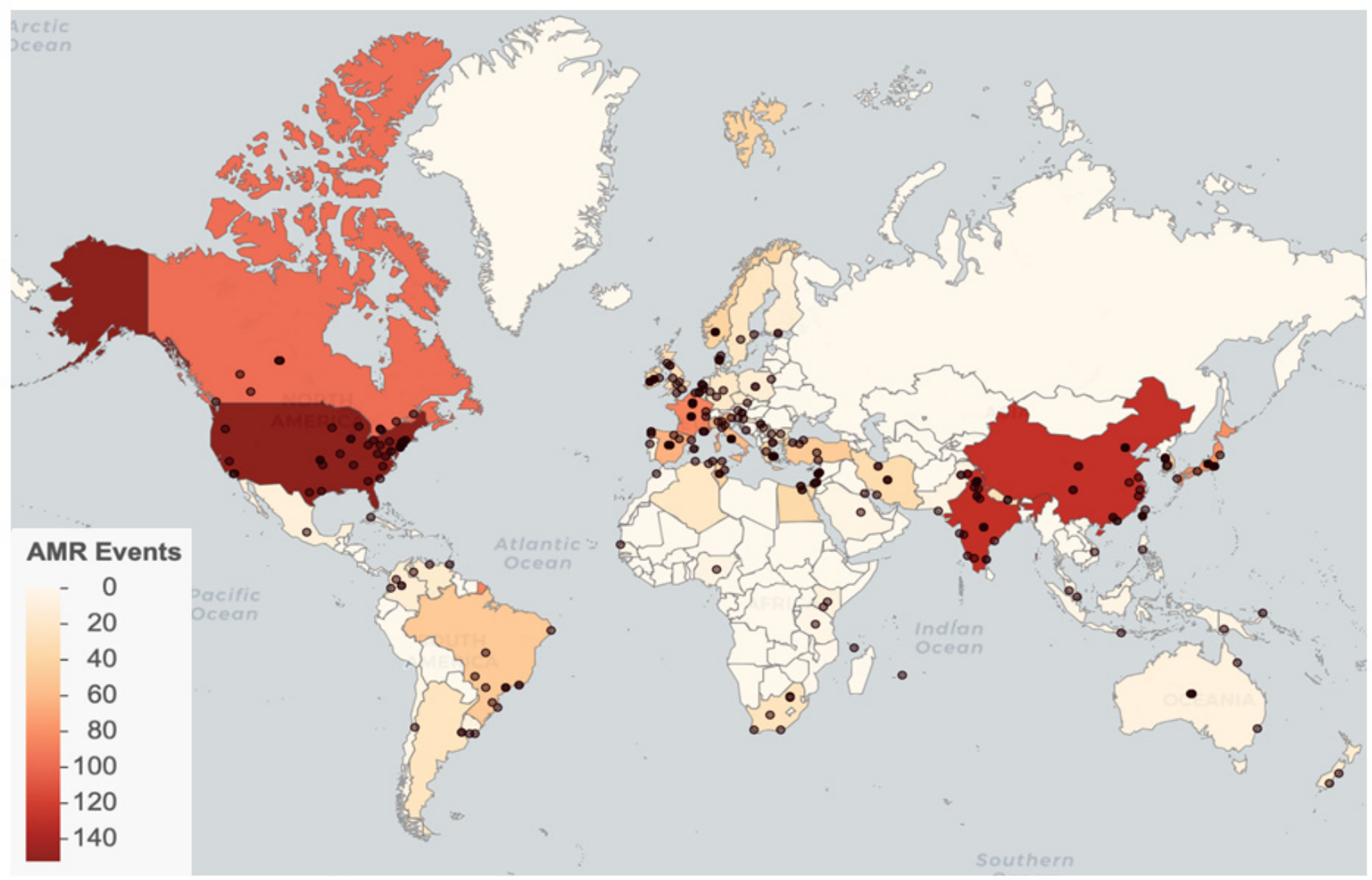

Figure 2. Global distribution of antimicrobial resistance (AMR) emergence events. Points represent locations. Countries are shaded by event count.

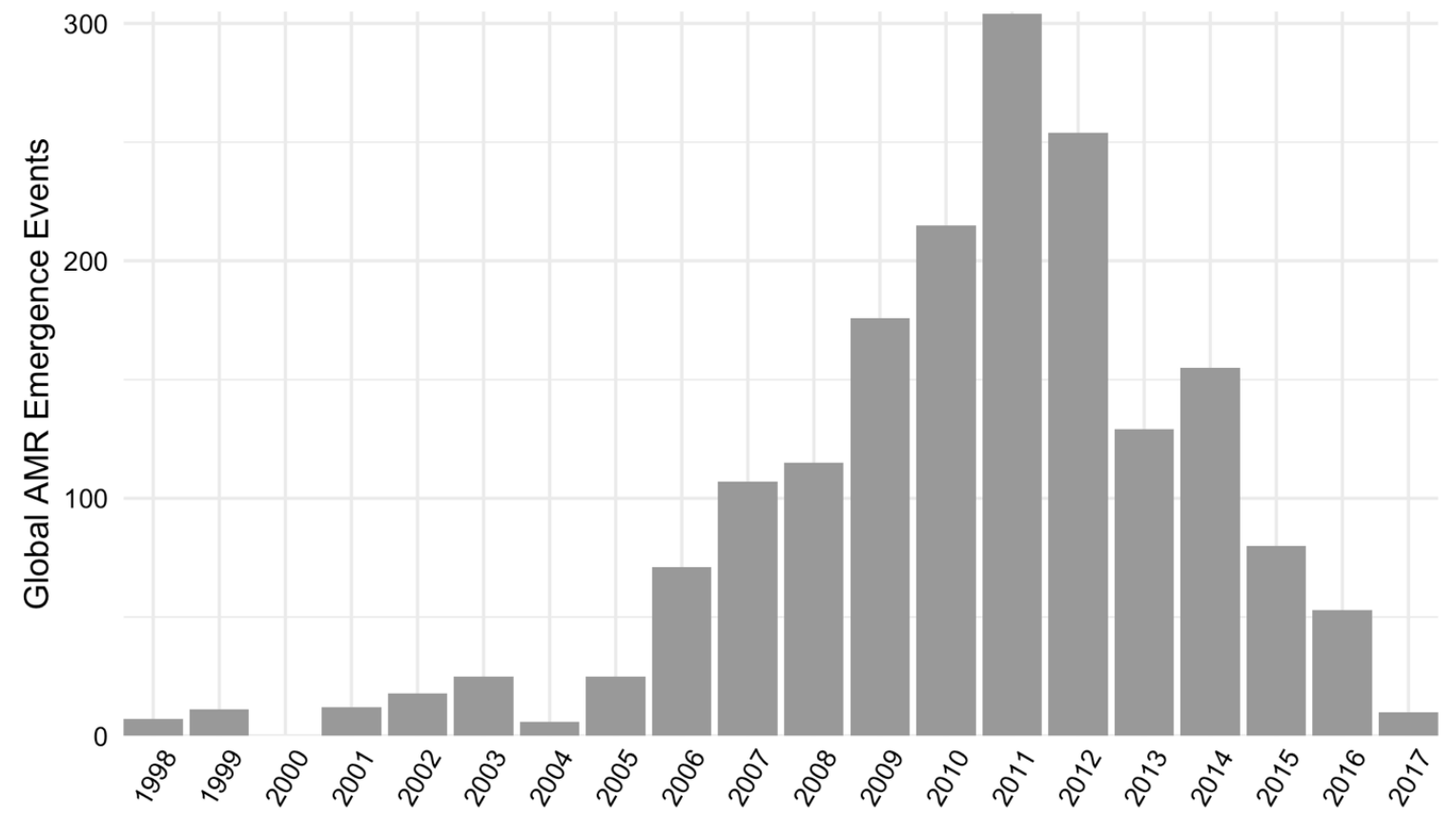

Figure 3. Global number of antimicrobial resistance (AMR) emergence events in the database disaggregated by year. 


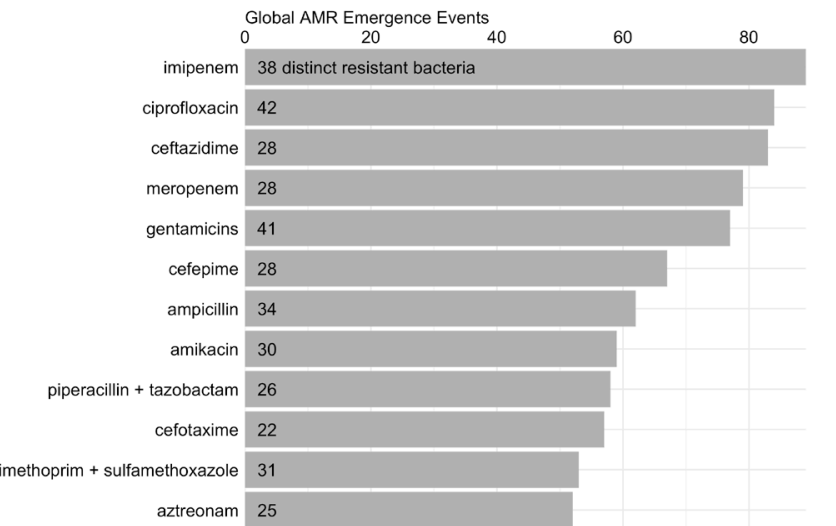

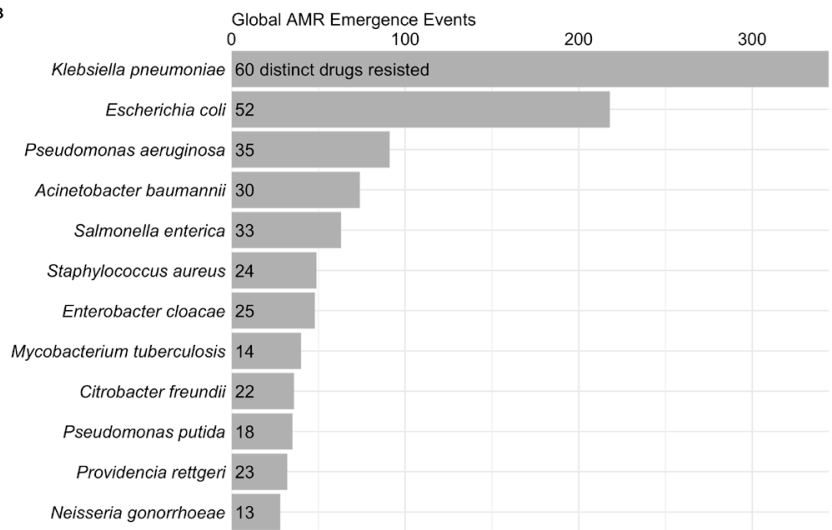

\begin{tabular}{|c|c|c|c|c|c|c|c|c|c|c|c|c|}
\hline & $\begin{array}{c}\text { Klebsiella } \\
\text { pneumoniae }\end{array}$ & $\begin{array}{c}\text { Escherichia } \\
\text { coli }\end{array}$ & $\begin{array}{c}\text { Pseudomonas } \\
\text { aeruginosa }\end{array}$ & $\begin{array}{c}\text { Acinetobacter } \\
\text { baumannii }\end{array}$ & $\begin{array}{c}\text { Salmonella } \\
\text { enterica }\end{array}$ & $\begin{array}{l}\text { Staphylococcus } \\
\text { aureus }\end{array}$ & $\begin{array}{c}\text { Enterobacter } \\
\text { cloacae }\end{array}$ & $\begin{array}{l}\text { Mycobacterium } \\
\text { tuberculosis }\end{array}$ & $\begin{array}{l}\text { Citrobacter } \\
\text { freundii }\end{array}$ & $\begin{array}{l}\text { Pseudomonas } \\
\text { putida }\end{array}$ & $\begin{array}{c}\text { Providencia } \\
\text { rettgeri }\end{array}$ & $\begin{array}{c}\text { Neisseria } \\
\text { gonorrhoeae }\end{array}$ \\
\hline imipenem & 22 & 14 & 5 & 7 & 2 & & 1 & & 1 & 3 & 2 & \\
\hline ciprofloxacin & 16 & 5 & 7 & 6 & 2 & 2 & 2 & & 1 & 3 & 2 & 6 \\
\hline ceftazidime & 19 & 12 & 7 & 6 & 6 & 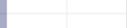 & 3 & & 4 & 3 & 2 & \\
\hline meropenem & 23 & 14 & 5 & 7 & 1 & 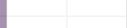 & 1 & & 1 & 3 & 2 & \\
\hline gentamicins & 10 & 8 & 5 & 4 & 4 & 4 & 3 & & & 3 & 2 & \\
\hline cefepime & 14 & 8 & 8 & 6 & 1 & 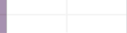 & 2 & & 4 & 3 & 2 & \\
\hline ampicillin & 8 & 9 & 1 & 1 & 5 & 1 & 1 & & 4 & - & 1 & \\
\hline amikacin & 11 & 7 & 5 & 5 & & 1 & 2 & 4 & & 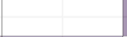 & 2 & \\
\hline piperacillin + tazobactam & 14 & 9 & 6 & 1 & 2 & 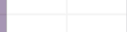 & 4 & & 1 & 3 & & \\
\hline cefotaxime & 16 & 11 & 1 & 1 & 6 & 1 & 2 & & 3 & 1 & & 1 \\
\hline trimethoprim + sulfamethoxazole & 9 & 5 & 3 & 2 & 2 & 1 & 3 & & & 1 & & \\
\hline aztreonam & 13 & 8 & 4 & 2 & 2 & & 2 & & 2 & 2 & 1 & \\
\hline
\end{tabular}

Figure 4. Global antimicrobial resistance (AMR) emergence events for top 12 drugs (a) and bacteria (b), and for their combinations (c). Counts within bars represent distinct number of resistant bacteria (a) and drugs (b).

Temporal patterns of AMR emergence events in the database reflect potentially incomplete coverage prior to 2006, as only ProMED-mail (not PubMed or Embase) was searched for this period. Further, the decrease in emergence events following the peak in 2011 likely reflects, in part, lags between event occurrence and reporting. Continued updating of the database is needed to determine long-term temporal trends.

There are several sources of ambiguity in the dataset due to imprecise reporting in literature (Table 2). While most studies reported the exact city or hospital of the emergence event $(n=200)$, others reported only state/province $(n=21)$ or study country $(\mathrm{n}=73)$. Differences in geographic scales would need to be considered if using this dataset for spatial analysis. In addition, there are inconsistencies in studies' reporting of drug and bacteria names. While most studies reported specific drug names $(n=83)$, others reported broad classes of drugs $(\mathrm{n}=32)$. Study authors that reported classes of drugs may have administered multiple drugs to the patient(s), but because the specific drugs were not reported, we were only able to count the drug as part of a single event. In addition, some drugs may be been administered as part of a mixture whereas other drugs were administered independently of each other (e.g., following failure of previous drug to treat infection).
Table 2. Classification specificity of four primary database fields.

\begin{tabular}{|c|c|c|}
\hline \multirow{2}{*}{ Drug } & Classification & Count \\
\hline \multirow{2}{*}{ drug name } & 83 \\
\hline drug group & 32 \\
\hline \multirow{2}{*}{ Bacteria } & drug group/name combo & 3 \\
\hline \multirow{2}{*}{ species } & 106 \\
\hline \multirow{2}{*}{ Location } & genus & 4 \\
\hline \multirow{2}{*}{} & family & 1 \\
\hline & hospital & 123 \\
\hline \multirow{2}{*}{ Start Date } & city & 77 \\
\hline & country & 21 \\
\hline & day & 73 \\
\hline & month & 121 \\
\hline
\end{tabular}


Due to ambiguity in reporting, we were not able to parse out mixtures versus independent drugs, and therefore treated each drug reported as independent. Bacteria were primarily reported to the species level $(n=106)$, but some were reported only to the genus or family level $(n=5)$. In these cases, it is possible that the species was novel or not identifiable. Many studies that reported bacterial species also reported strains, and it is possible that there are differences in resistance by strain. However, due to inconsistent reporting, we have not standardized reported strains.

This database presents first AMR emergence events aggregated at the country level. Emergence events may be due to either local mutation events or imported resistance from other geographies. Future analyses examining the underlying determinants of first emergence at a country level should consider import as a possible mechanism. For example, some studies reported locations of residence and recent travel of patients, and where available we have included this information, which may support such analysis. Analyses only examining first global emergence events should remove records of all but the earliest dates of each unique bacteria-drug combination.

\section{Data availability}

Zenodo: A global repository of novel antimicrobial emergence events. http://doi.org/10.5281/zenodo. $4924992^{10}$.
This project contains the following underlying data:

- 'events-db.csv` (AMR emergence events database)

- 'data-processed/articles-db.csv' (Metadata about each article in the database, including citation information and full abstracts. This file can be joined with `events-db.csv by the `study_id` field.)

- `screening/ directory (All abstracts and ProMED-mail reports that were screened for potential inclusion in the database)

- 'data-raw/coded-segments` (raw exports from coded full-text articles)

- 'data-processed/segments.csv' (A pre-filtered, pretransformed database that contains all fields ad events)

License: MIT

\section{Code availability}

Source code available from: https://github.com/ecohealthalliance/ amr-db

Archived source code at time of publication: http://doi.org/ 10.5281 /zenodo. $4924992^{10}$

License: MIT
1. World Health Organization: Global action plan on antimicrobial resistance. 2015. Reference Source

2. Cosgrove SE: The relationship between antimicrobial resistance and patient outcomes: mortality, length of hospital stay, and health care costs. Clin Infect Dis. 2006; 42 Suppl 2: S82-89. PubMed Abstract | Publisher Full Text

3. World Health Organization: Antimicrobial resistance: global report on surveillance 2014. 2014 Reference Source

4. Byarugaba DK: A view on antimicrobial resistance in developing countries and responsible risk factors. Int J Antimicrob Agents. 2004; 24(2): 105-110. PubMed Abstract | Publisher Full Text

5. Morgan DJ, Okeke IN, Laxminarayan R, et al.: Non-prescription antimicrobial use worldwide: a systematic review. Lancet Infect Dis. 2011; 11(9): 692-701. PubMed Abstract | Publisher Full Text | Free Full Text

6. Collignon P: The importance of a One Health approach to preventing the development and spread of antibiotic resistance. Curr Top Microbiol Immunol. 2013; 366: 19-36. PubMed Abstract

7. Interagency Coordination Group on Antimicrobial Resistance: No Time to Wait: Securing the Future from Drug-Resistant Infections. 2019. Reference Source

8. ResistanceMap: Center for Disease Dynamics, Economics \& Policy. 2017 Reference Source

9. Lajeunesse MJ: Facilitating systematic reviews, data extraction and metaanalysis with the metagear package for R. Methods in Ecology and Evolution. 2016; 7(3): 323-330. Publisher Full Text

10. Mendelsohn E, Ross N, White A, et al:: A global repository of novel antimicrobial emergence events (Version 2.0.0). Zenodo. 2020. http://www.doi.org/10.5281/zenodo.4924992

11. VERBI Software, MAXQDA 2018. 2017. Reference Source
12. Duckles B, Sholler D, Draper J, et al.: qcoder: Lightweight Qualitative Coding. R package version 0.1.0. 2020 . Reference Source

13. National Library of Medicine: Medical Subject Headings. 2020. Reference Source

14. National Library of Medicine: National Center for Biotechnology Information NCBI) Organismal Classification. 2012 Reference Source

15. Whetzel PL, Noy NF, Shah NH, et al:: BioPortal: enhanced functionality via new Web services from the National Center for Biomedical Ontology to access and use ontologies in software applications. Nucleic Acids Res. 2011; 39(Web Server issue): W541-545. PubMed Abstract | Publisher Full Text | Free Full Text

16. World Health Organization Collaborating Centre for Drug Statistics Methodology: Anatomical Therapeutic Chemical Classification. 2020. Reference Source

17. R Core Team: R Foundation for Statistical Computing. Vienna, Austria. 2019 Reference Source

18. Wickham HAM, Bryan J, Chang W, et al.: Welcome to the tidyverse. J Open Source Softw. 2019; 4: 1686. Publisher Full Text

19. Kahle $D$, Wickham $\mathrm{H}$ : ggmap: Spatial Visualization with ggplot2. The $R$ Journal. 2013; 5: 144-161. Reference Source

20. Grolemund G, Wickham H: Dates and Times Made Easy with \{lubridate\}. J Stat Softw. 2011; 40: 1-25. Publisher Full Text

21. Codjoe FS, Donkor ES: Carbapenem Resistance: A Review. Med Sci (Basel). 2017; 6(1): 1. PubMed Abstract | Publisher Full Text | Free Full Text

22. World Health Organization: Citically Important Antimicrobials for Human Medicine. 2016. Reference Source 
23. Paterson DL, Harris PNA: Colistin resistance: a major breach in our last line of defence. Lancet Infect Dis. 2016; 16(2): 132-133. PubMed Abstract | Publisher Full Text

24. Berthe $F$, Cesar J, Bouley $T$, et al.: Operational framework for strengthening human, animal and environmental public health systems at their interface (English). Washington, D.C.: World Bank Group. 2018.

Reference Source
25. Vanlangendonck C, Mackenzie J, Osterhaus A: Highlights from Science Policy Interface sessions at the One Health Congress 2020. One Health Outlook. 2021; 3(1): 1

$2021 ; 3(1): 1$.
PubMed Abstract | Publisher Full Text | Free Full Text

26. Konno K, Akasaka M, Koshida C, et al.: Ignoring non-English-language studies may bias ecological meta-analyses. Ecol Evol. 2020; 10(13): 6373-6384.

PubMed Abstract | Publisher Full Text | Free Full Text 


\section{Open Peer Review}

\section{Current Peer Review Status: $\checkmark$ ? ?}

\section{Version 2}

Reviewer Report 16 November 2021

https://doi.org/10.5256/f1000research.57747.r99191

(C) 2021 Pinto Ferreira J. This is an open access peer review report distributed under the terms of the Creative Commons Attribution License, which permits unrestricted use, distribution, and reproduction in any medium, provided the original work is properly cited.

\section{? Jorge Pinto Ferreira (i)}

Antimicrobial Resistance and Veterinary Products Department, World Organisation for Animal Health, Paris, France

1. The title needs to reflect that the paper only focus on humans.

2. "Novel" in this case only refers to "novel in the country" - this is a major characteristic, that needs to be emphasized, if possible, even in the title.

3. In the introduction, the reference to IACG does not make sense any more, as the IACG is no longer existent. It lead to a couple of new structures, including, for example, the Global Leaders Group, and these should be mentioned in the paper.

4. Fig. 3: even if speculative, the peaks between 2010-2012 should be explained.

Is the rationale for creating the dataset(s) clearly described?

Yes

Are the protocols appropriate and is the work technically sound?

Yes

Are sufficient details of methods and materials provided to allow replication by others?

Yes

Are the datasets clearly presented in a useable and accessible format?

Yes

Competing Interests: No competing interests were disclosed.

Reviewer Expertise: AMR, AMU, policy 
I confirm that I have read this submission and believe that I have an appropriate level of expertise to confirm that it is of an acceptable scientific standard, however I have significant reservations, as outlined above.

Reviewer Report 09 August 2021

https://doi.org/10.5256/f1000research.57747.r87972

(C) 2021 Eremin S. This is an open access peer review report distributed under the terms of the Creative Commons Attribution License, which permits unrestricted use, distribution, and reproduction in any medium, provided the original work is properly cited.

\section{Sergey Eremin}

World Health Organization, Geneva, Switzerland

We have no further comments to make.

Is the rationale for creating the dataset(s) clearly described?

Yes

Are the protocols appropriate and is the work technically sound?

Yes

Are sufficient details of methods and materials provided to allow replication by others? Yes

Are the datasets clearly presented in a useable and accessible format?

Yes

Competing Interests: No competing interests were disclosed.

Reviewer Expertise: Surveillance of antimicrobial resistance.

I confirm that I have read this submission and believe that I have an appropriate level of expertise to confirm that it is of an acceptable scientific standard.

\section{Version 1}

Reviewer Report 15 February 2021

https://doi.org/10.5256/f1000research.29674.r78874 
(c) 2021 Stobberingh $\mathbf{E}$. This is an open access peer review report distributed under the terms of the Creative Commons Attribution License, which permits unrestricted use, distribution, and reproduction in any medium, provided the original work is properly cited.

\section{Ellen Stobberingh}

Care And Public Health Research Institute (CAPHRI), Maastricht University Medical Centre (MUMC), Maastricht, The Netherlands

The authors analysed the prevalence of novel AMR in humans in the country. Several questions need to be clarified:

- Inclusion criteria:

AMR in asymptomatic commensals were not included as these do not meet the inclusion criteria of clinical cases. However, resistance in commensals is frequently the precursor of resistance in clincal isolates. Excluding the commensals might contribute to lower prevalence and later report of resistance.

Focus on humans only did not address the One health approach which is especially relevant in AMR because of the spread from animals to humans. This should be added in the discussion.

Novel: was defined as novel for that country. But the resistance might be described already in other countries. Transfer from these countries for instance via travel might contribute to spread of these AMR to other countries and is not a novel resistance. Pick up of AMR resistance during travel starts mostly as a commensal and later on possible as infection. Please comment.

Resistance: defined as to survive AMR treatment, i.e. the bacteria is able to continue to cause disease in the host or to spread to others for a longer than the standard period as determined by the WHO or the national guidelines. What to do in case of discrepancy between these two?

Interscore $82 \%$ in case of discrepancy the decision of a third reviewer was decisive?

What is the possible explanation for the highest prevalence in $2011 ?$

Resistance to carbapenems as last resort, colistin when no other options are available. This suggests a discrepancy, please rephrase.

Is the rationale for creating the dataset(s) clearly described?

Partly

Are the protocols appropriate and is the work technically sound? Partly

Are sufficient details of methods and materials provided to allow replication by others? Yes

Are the datasets clearly presented in a useable and accessible format? 
Yes

Competing Interests: No competing interests were disclosed.

Reviewer Expertise: Antimicrobial resistance prevalence and spread, Bacteriology.

I confirm that I have read this submission and believe that I have an appropriate level of expertise to confirm that it is of an acceptable scientific standard, however I have significant reservations, as outlined above.

Author Response 10 Jun 2021

Emma Mendelsohn, EcoHealth Alliance, New York, USA

Thank you for your thorough review and comments (especially appreciate your thoughts on the One Health context and potential spread mechanisms). We address your comments below.

AMR in asymptomatic commensals were not included as these do not meet the inclusion criteria of clinical cases. However, resistance in commensals is frequently the precursor of resistance in clincal isolates. Excluding the commensals might contribute to lower prevalence and later report of resistance.

We agree that asymptomatic commensals are important to identifying resistance before clinical emergence. However, we did not include commensals in the database as we found there is high variability in study design among these studies (e.g., drugs tested). Clinical reports, while still subject to variability and bias, tend to be more consistent in design (reporting only on resistances observed with illness). We address this concern in the Abstract and report screening section of the manuscript.

Focus on humans only did not address the One health approach which is especially relevant in AMR because of the spread from animals to humans. This should be added in the discussion.

This is a good point and we have added text about potential risk factors (including livestock) for emergence and the relevance to One Health in the discussion section.

Novel: was defined as novel for that country. But the resistance might be described already in other countries. Transfer from these countries for instance via travel might contribute to spread of these AMR to other countries and is not a novel resistance. Pick up of AMR resistance during travel starts mostly as a commensal and later on possible as infection. Please comment.

We agree and note that emergence events within a country may be due to mutation or transport from other geographies. For first global emergence events, users of the database should filter to the first occurrence of each bacteria-drug combination. We have added discussion about travel and migration being potential risk factors for emergence in countries. 
Resistance: defined as to survive AMR treatment, i.e. the bacteria is able to continue to cause disease in the host or to spread to others for a longer than the standard period as determined by the WHO or the national guidelines. What to do in case of discrepancy between these two?

As most authors did not report which guidelines they used, we deferred to the authors selection of protocols and did not evaluate any potential discrepancies. We have added text to clarify this.

Interscore $\mathbf{8 2} \%$ in case of discrepancy the decision of a third reviewer was decisive?

Yes; we have rephrased to clarify this.

What is the possible explanation for the highest prevalence in 2011?

We have rephrased the text in Data usage notes to clarify that the peak in 2011 is likely an effect of reporting lag for more recent events.

Resistance to carbapenems as last resort, colistin when no other options are available. This suggests a discrepancy, please rephrase.

Good catch. We have rephrased.

Competing Interests: No competing interests were disclosed.

Reviewer Report 07 December 2020

https://doi.org/10.5256/f1000research.29674.r75116

(C) 2020 Eremin S et al. This is an open access peer review report distributed under the terms of the Creative Commons Attribution License, which permits unrestricted use, distribution, and reproduction in any medium, provided the original work is properly cited.

\section{Barbara Tornimbene}

World Health Organization, Geneva, Switzerland

Sergey Eremin

World Health Organization, Geneva, Switzerland

The paper represents an impressive effort to fill in the gaps in our understanding of emergence and spread of antimicrobial resistance in bacterial pathogens and provides results of a systematic review of English-language scientific literature and surveillance reports to identify the spatial and temporal patterns of the emerging AMR.

Is the rationale for creating the dataset(s) clearly described? 
Although the author rationale for generating a publicly available source of temporal and spatial data for emerging resistance is clear, it could be better explained in the title and the introduction that the creating of a database is the objective of the study. The authors could also go into more detail on how the database will support on-going agreements and programs, particularly by defining on-going agreements. It would be also important to clarify what will be the role of the government of countries that appear in the dataset, in terms of acknowledging and validating published data.

\section{Are the protocols appropriate and is the work technically sound?}

Merging of results originating from peer-reviewed literature, which undergoes a strict scientific scrutiny, and data generated by ProMED-mail, which does not follow the same rigour, can be exposed, particularly when reporting novel events, to high level of inconsistency. No distinction between study designs screened in the literature was applied and results adjusted accordingly. The exclusion criteria could be expanded. For example, no assessment of the quality and reliability of these studies was done before including them in the final pool of articles.

The search strategy may benefit from inclusion of terms related to "drug resistance", the term used in quite a number of reports instead of "antibiotic resistance" or "antimicrobial resistance", especially when reporting infections caused by MDR or XDR or PDR pathogens.

Finally, the authors mentioned in the "Database usage notes" section that the reported events represent a limited subset of information and strongly reflect reporting effort and practices. Other limitations are also listed in this section. This should probably be part of the discussion and better reflected on the abstract when listing the most common bacteria demonstrating new resistance. Moreover, an in-depth discussion on the impact of these bias should be added, particularly the effect that variability in reporting effort and practices, and quality and reliability of the studies, have on obtained results.

\section{Are sufficient details of methods and materials provided to allow replication by others?} Some definitions in the inclusion criteria are not sufficiently clear.

A clinical case is defined as "an individual who presents with symptoms to a medical professional and is determined by a medical professional to have been infected with the bacterium in question". This sounds rather vague and we believe that the authors could be more consistent when defining "bacterium in question" or "bacterium of interest". This is important to understand how cases were included.

The definition of novel resistance seems to be uncertain. It has to be clearly explained in the paper why only phenotypic test results were chosen and why only specific drug-bug combinations are considered and not emergence of certain types of multidrug resistance. The authors might also want to clarify why detection of a novel gene responsible, using example given in the paper, for production of a new type of beta-lactamase or, specifically, carbapenemase, is not considered novel and should be ignored. Such an event has to be detected and followed as it may have a significant impact on treatment options and other public health implications. Again, more details should be given on how the "novelty" was identified when reviewing the papers. For example, the paper coded 15378 in the database describes both phenotypic and molecular resistance testing results obtained from 12 clinical isolates from 12 patients with infections caused by Enterococcus faecium and 11 supposedly novel resistance events were included in the database. But the only 
event that was defined by the authors of the report as novel was the first detection in the country of the clonal complex 17 VREF. Resistance to e.g. clindamycin, gentamicin, and several other antibiotics in all reported isolates could hardly be considered novel or emerging.

The authors should better explain the added value of including the "drug_parent_name" variable with the names of the taxonomic parent of antimicrobial drug, standardized to the MeSH ontology. While it may be reflecting the methodology of the database creation, using a classification more suited to the field of study, such as e.g. the Anatomical Therapeutic Chemical (ATC) classification system may be considered.

Finally, as the main goal of the database is to present temporal and spatial data, a better definition of temporal data should be given. In the paper the authors mention "event date", "start date", "study date" and "end date". Aside the lack of consistency it should be clearer if the authors are considering the date the patients started experiencing symptoms, the date the patients was diagnosed, the date the diagnosis results were obtained, or the date the study started. This is key to allow replication by others.

Are the datasets clearly presented in a useable and accessible format?

The detailed dataset is easily accessible and could be downloaded but is not sufficiently userfriendly. Using it would require certain data management skills from the end-users and therefore might limit their number.

Is the rationale for creating the dataset(s) clearly described?

Partly

Are the protocols appropriate and is the work technically sound? Partly

Are sufficient details of methods and materials provided to allow replication by others? Partly

Are the datasets clearly presented in a useable and accessible format?

Partly

Competing Interests: No competing interests were disclosed.

Reviewer Expertise: Surveillance of antimicrobial resistance.

We confirm that we have read this submission and believe that we have an appropriate level of expertise to confirm that it is of an acceptable scientific standard, however we have significant reservations, as outlined above.

Author Response 10 Jun 2021

Emma Mendelsohn, EcoHealth Alliance, New York, USA

Thank you for your thorough review. We appreciate your attention to detail and thoughtful 
comments, which we address below.

Is the rationale for creating the dataset(s) clearly described?

Although the author rationale for generating a publicly available source of temporal and spatial data for emerging resistance is clear, it could be better explained in the title and the introduction that the creating of a database is the objective of the study. The authors could also go into more detail on how the database will support on-going agreements and programs, particularly by defining on-going agreements. It would be also important to clarify what will be the role of the government of countries that appear in the dataset, in terms of acknowledging and validating published data.

We agree and have emphasized the database as a study objective by changing the title to " $\mathrm{A}$ global repository of novel antimicrobial emergence events". We also added details on the rationale and main goals of the paper in the introduction, highlighting the relevance of this dataset in supporting on-going international agreements and programs.

Are the protocols appropriate and is the work technically sound?

Merging of results originating from peer-reviewed literature, which undergoes a strict scientific scrutiny, and data generated by ProMED-mail, which does not follow the same rigour, can be exposed, particularly when reporting novel events, to high level of inconsistency. No distinction between study designs screened in the literature was applied and results adjusted accordingly. The exclusion criteria could be expanded. For example, no assessment of the quality and reliability of these studies was done before including them in the final pool of articles.

Thank you for the relevant comment. This is an important point to be considered by the end-users. We have clarified in the manuscript that we did not conduct an assessment of study/report quality and have described this as a source of uncertainty. In addition, we have added a field to the database indicating whether an event is from a published study or from ProMED-mail, and we include in the text summarized counts of events by source type.

The search strategy may benefit from inclusion of terms related to "drug resistance", the term used in quite a number of reports instead of "antibiotic resistance" or "antimicrobial resistance", especially when reporting infections caused by MDR or XDR or PDR pathogens.

We agree that "drug resistance" may yield relevant results. Unfortunately we are unable to redo the existing literature search at this time due to the high level of effort required and additional human resources not available at this time. We will include this term in future iterations of the project.

Finally, the authors mentioned in the "Database usage notes" section that the reported events represent a limited subset of information and strongly reflect reporting effort and practices. Other limitations are also listed in this section. This should probably be part of the discussion and better reflected on the abstract when listing the most common bacteria demonstrating new resistance. Moreover, an indepth discussion on the impact of these bias should be added, particularly the effect 
that variability in reporting effort and practices, and quality and reliability of the studies, have on obtained results.

We thank the reviewer for the suggestion. Bias in reporting effort is indeed a recurrent problem in surveillance. We have added mention of reporting biases to the abstract and discussion section (referring readers to Database usage notes for further detail). We also now address quality of studies as a source of uncertainty in the database.

Are sufficient details of methods and materials provided to allow replication by others?

Some definitions in the inclusion criteria are not sufficiently clear.

A clinical case is defined as "an individual who presents with symptoms to a medical professional and is determined by a medical professional to have been infected with the bacterium in question". This sounds rather vague and we believe that the authors could be more consistent when defining "bacterium in question" or "bacterium of interest". This is important to understand how cases were included.

We adjusted the wording to clarify that bacteria is any species of pathogen in the domain Bacteria, and have replaced the term "bacterium in question" with "bacterium species reported".

Due to inconsistencies in reporting, we were unable to consistently identify pathogens by strain. However, this data is available as an unstandardized field in the database and we added.

The definition of novel resistance seems to be uncertain. It has to be clearly explained in the paper why only phenotypic test results were chosen and why only specific drugbug combinations are considered and not emergence of certain types of multidrug resistance. The authors might also want to clarify why detection of a novel gene responsible, using example given in the paper, for production of a new type of betalactamase or, specifically, carbapenemase, is not considered novel and should be ignored. Such an event has to be detected and followed as it may have a significant impact on treatment options and other public health implications. Again, more details should be given on how the "novelty" was identified when reviewing the papers. For example, the paper coded 15378 in the database describes both phenotypic and molecular resistance testing results obtained from 12 clinical isolates from 12 patients with infections caused by Enterococcus faecium and 11 supposedly novel resistance events were included in the database. But the only event that was defined by the authors of the report as novel was the first detection in the country of the clonal complex 17 VREF. Resistance to e.g. clindamycin, gentamicin, and several other antibiotics in all reported isolates could hardly be considered novel or emerging.

We agree this is a source of uncertainty in the database. In response to your comment, we have revisited the drug name standardization and disaggregated all drug combinations, as we had not been consistent in identifying combinations. We are now treating all drugs as being separately administered. Unfortunately, due to inconsistencies in reporting, we are 
not able to effectively characterize which drugs were administered independently and which were administered as part of a complex. We address this uncertainty in the Database usage notes.

The authors should better explain the added value of including the "drug_parent_name" variable with the names of the taxonomic parent of antimicrobial drug, standardized to the MeSH ontology. While it may be reflecting the methodology of the database creation, using a classification more suited to the field of study, such as e.g. the Anatomical Therapeutic Chemical (ATC) classification system may be considered.

Based on your comments we explored standardizing drug names to ATC. We found that results were very similar to MeSH (i.e., counts changed only slightly). We discuss this in the manuscript and have added an alternate version of the database with ATC standardization to the project repository.

Finally, as the main goal of the database is to present temporal and spatial data, a better definition of temporal data should be given. In the paper the authors mention "event date", "start date", "study date" and "end date". Aside the lack of consistency it should be clearer if the authors are considering the date the patients started experiencing symptoms, the date the patients was diagnosed, the date the diagnosis results were obtained, or the date the study started. This is key to allow replication by others.

We switched to consistent usage of "start date" and clarified that it refers to the date the patient presented to the hospital or clinic.

Are the datasets clearly presented in a useable and accessible format? The detailed dataset is easily accessible and could be downloaded but is not sufficiently user-friendly. Using it would require certain data management skills from the end-users and therefore might limit their number.

We added a sentence to clarify that the database is available for download as a single csv file ('events-db.csv')

Competing Interests: No competing interests were disclosed. 
The benefits of publishing with F1000Research:

- Your article is published within days, with no editorial bias

- You can publish traditional articles, null/negative results, case reports, data notes and more

- The peer review process is transparent and collaborative

- Your article is indexed in PubMed after passing peer review

- Dedicated customer support at every stage

For pre-submission enquiries, contact research@f1000.com 